\title{
Design of the Structure of 1-3 Piezocomposites by Considering the Interpillar Vibrational Modes
}

\author{
Seonghun Pyo and Yongrae Roh* \\ School of Mechanical Engineering, Kyungpook National University, \\ 80 Daehakro, Bukgu, Daegu 41566, Korea
}

(Received September 14, 2015; accepted January 18, 2016)

Keywords: interpillar vibrational mode, optimization, 1-3 piezocomposites, finite element analysis, thickness-mode coupling coefficient

When a piezocomposite vibrates in a thickness mode, interpillar modes of vibration are likely to occur between lattice-structured piezoceramic pillars and a polymer matrix, which significantly diminishes the performance of the piezocomposite. The conventional method for designing 1-3 piezocomposite structures has not considered these interpillar modes. In this study, a new method of optimizing the structure of 1-3 piezocomposites has been developed to maximize the electromechanical coupling factor of thickness modes while preventing the occurrence of interpillar resonant modes. The volume fraction of piezoceramics and the aspect ratio of the piezocomposite were the design variables that were optimized. The genetic algorithm was used to optimize the structure, and the finite element method was used to analyze the interpillar modes.

\section{Introduction}

1-3 piezocomposites have a lattice structure composed of piezoceramic pillars embedded in a polymer matrix. Figure 1 shows a schematic diagram of the structure of a typical 1-3 piezocomposite. 1-3 piezocomposites have been widely used in various fields to overcome the limitations of conventional bulk piezoceramics. ${ }^{(1,2)}$ In particular, the high electromechanical coupling coefficient, high hydrostatic figure of merit, and low acoustic impedance of 1-3 piezocomposites are advantageous properties for underwater applications. ${ }^{(3-7)}$

The characteristics of piezocomposites and the method of designing their structure have been extensively studied by various research groups. Such studies have served as prominent reference points for the development and fabrication of 1-3 piezocomposites. ${ }^{(1-4,8-13)}$ Gururaja et $a l .^{(1)}$ studied the vibrational modes of piezocomposites in relation to the ceramic volume fraction $(V F)$ and thickness of the piezocomposites. Newnham ${ }^{(2)}$ investigated the thickness and lateral vibrational modes of piezocomposites. In addition, Newnham et al. ${ }^{(4)}$ studied the characteristics of piezocomposites with respect to their connectivity patterns. Smith and coworker, ${ }^{(10,11)}$ as well as the authors of the present paper, ${ }^{(12)}$ studied the equivalent-material properties of piezocomposites. Gibiansky and Torquato $^{(9)}$ investigated the optimization of the structure of piezocomposites in terms of the cross-sectional shape and array pattern of piezoceramic pillars, the $V F$ of each constituent, and properties of the polymer matrix to apply the piezocomposites as a hydrophone. However,

"Corresponding author: e-mail: yryong@knu.ac.kr 


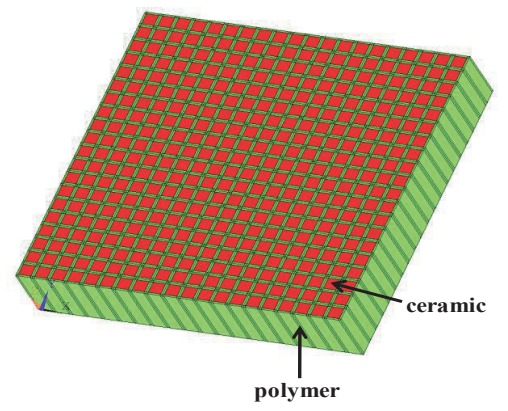

Fig. 1. (Color online) Schematic of the structure of a typical 1-3 piezocomposite.

these investigations of the structural design of 1-3 piezocomposites have focused on increasing the piezoelectric activity of the piezocomposites by assuming that 1-3 piezocomposites only operate in pure thickness modes.

It is important for the piezocomposite to operate stably in pure thickness modes near its series resonance with proper compensation of temperature and other environmental influences. ${ }^{(14-18)}$ However, in practice, when a 1-3 piezocomposite vibrates in a thickness mode, it is likely that interpillar resonant modes will be generated by the lateral waves reflected at the interface between lattice-structured piezoceramic pillars and a polymer matrix, which significantly diminishes the performance of the piezocomposite. The operating mechanism of such interpillar resonant modes has been studied by many researchers. ${ }^{(19-21)}$ When a thickness mode is coupled to an interpillar mode, the deformation of the piezoceramic pillars becomes out of phase with that of the polymer matrix. Hence, the overall electromechanical coupling factor in the direction of the sample thickness declines, which results in the poor performance of acoustic transducers made with 1-3 piezocomposites. ${ }^{(22,23)}$ Conventional methods of designing 1-3 piezocomposite structures have not considered these interpillar modes, which motivated the work that is presented in this paper.

In this study, a new method of optimizing the structure of 1-3 piezocomposites was developed to maximize the electromechanical coupling factor for thickness modes while preventing the occurrence of interpillar resonant modes. The structural variables used to control the properties of the piezocomposites were the $V F$ and the aspect ratio $(A R)$ because these are the most easily controllable parameters in the fabrication process for piezocomposites. The piezoceramic $V F$ is defined as (piezoceramic pillar width) ${ }^{2} /\left(\right.$ piezoceramic pillar pitch) ${ }^{2}$, while $A R$ is defined as (piezoceramic pillar pitch)/(piezocomposite plate thickness). The genetic algorithm (GA) was used to optimize the structure, and the finite element (FE) method was used to analyze the interpillar modes. ${ }^{(24)}$

\section{Construction and Validation of the FE Model}

An FE model was constructed with the ANSYS ${ }^{\circledR}$ commercial software package to simulate the structure of a 1-3 piezocomposite plate, as shown in Fig. 1. The numbers of elements and nodes were 64000 and 72000, respectively. The piezoceramic and polymer matrix modeled were PZT$5 \mathrm{H}$ and a low-viscosity epoxy from Dow Corning Co. (Midland, MI, USA), respectively. The density, Poisson's ratio, and elastic modulus of the polymer matrix were set at $1120 \mathrm{~kg} / \mathrm{m}^{3}, 0.4$, and $4.34 \mathrm{GPa}$, respectively. The width of the plate specimen was $9.7 \mathrm{~mm}$. The thickness of the 
piezocomposite plate was $0.56 \mathrm{~mm}$, and the pitch of the piezoceramic pillars was $0.44 \mathrm{~mm}$, which increased the $A R$ to 0.79 . The width of the piezoceramic pillars was $0.22 \mathrm{~mm}$, and thus, $V F$ was $25 \%$. This FE model was used to analyze the characteristics of the piezocomposites, including the interpillar modes of vibration.

Before using the model, its validity was examined by fabricating the 1-3 piezocomposite plate and comparing the measured impedance spectrum with that calculated using the FE model. We fabricated the 1-3 piezocomposite specimen with the dice and fill method to produce a structure that was identical to the FE model. ${ }^{(25)}$ Figure 2 shows an image of the fabricated specimen. The impedance spectrum of the specimen was measured with an HP 4294A impedance analyzer (Agilent Technologies, Santa Clara, CA, USA). The measured spectrum was then compared with the calculated spectrum, as shown in Fig. 3. The electromechanical coupling factor of the thickness mode, $k_{\mathrm{t}}$, was determined to be 0.43 from the spectrum. The comparison shows that there is good agreement between the modeled and measured spectra, with the differences between the resonant and antiresonant frequencies being less than $2 \%$. These differences are partly due to the machining tolerance during the fabrication of the piezocomposite plate. In addition, it is believed that variations in the material properties of the polymer are also responsible for the differences.

The spectra show that the thickness mode of the piezocomposite plate is clearly coupled to the interpillar mode. The $A R$ of the specimen was purposely set to 0.79 to generate the interpillar modes. Therefore, the validity of the FE model for simulating the characteristics of 1-3 piezocomposites was confirmed, including the occurrence of interpillar modes, with a maximum difference of less than $2 \%$ when compared with the measurements.

\section{Analyzing the Effects of the Structure Variables}

Before designing the structure of the piezocomposites, we analyzed the effects of the structural variables on the occurrence of interpillar modes using the FE model. First, the impedance spectrum was analyzed as a function of $A R ; A R$ was varied from 0.3 to 0.6 at intervals of 0.1 , while $V F$ was

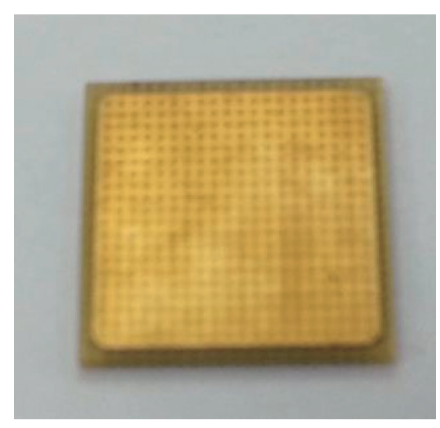

Fig. 2. (Color online) Fabricated 1-3 piezocomposite plate that was used to validate the FE model.

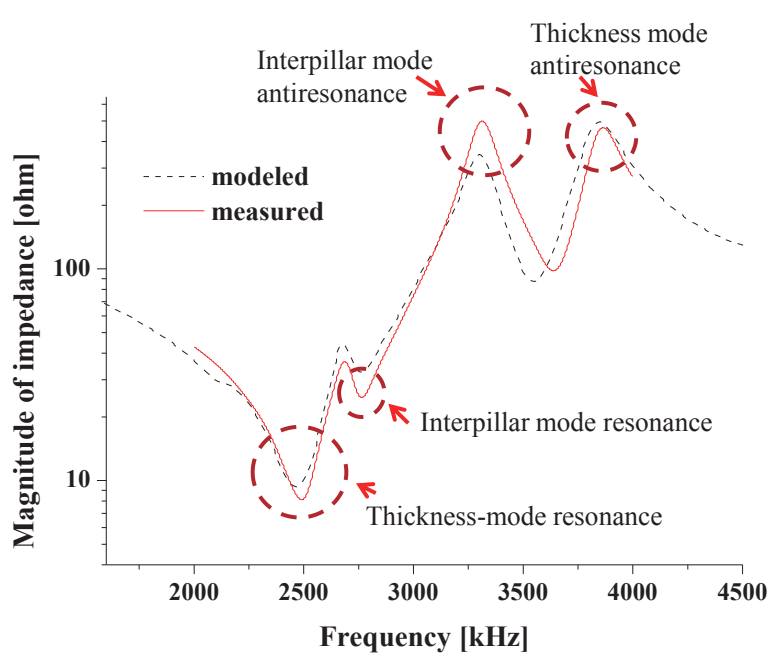

Fig. 3. (Color online) Comparison of the measured and modeled impedance spectra. 
kept constant at 20\%. Figure 4 shows that an interpillar mode occurs between the resonant and antiresonant frequencies of the thickness mode when $A R=0.6$. However, as $A R$ decreases, the interpillar mode moves to higher frequencies and becomes decoupled from the thickness mode. Hence, $A R$ should be small to prevent the occurrence of an interpillar mode. However, a small $A R$ produces a small kerf width between the piezoceramic pillars, which will increase the difficulty in fabricating such piezocomposites. Therefore, it is concluded that $A R$ must be as large as possible while keeping the interpillar mode uncoupled from the thickness mode of the 1-3 piezocomposite plate.

Next, the impedance spectrum was analyzed as a function of $V F$, as shown in Fig. 5. $V F$ was varied from 30 to $60 \%$ at intervals of $10 \%$, while $A R$ was maintained at 0.5 . The interpillar mode is coupled to the thickness mode when $V F=30$ and $40 \%$. However, as $V F$ increases, the interpillar mode is shifted to higher frequencies and becomes decoupled from the thickness mode. ${ }^{(21)}$ Hence, the piezoceramic $V F$ should be large to prevent the coupling of the interpillar mode. Increasing $V F$ and decreasing $A R$ have very similar effects on the interpillar mode, i.e., both lead to the narrowing of the gap between the piezoceramic pillars.

From the above analysis, it is concluded that the interpillar mode is likely to occur in the case of a small piezoceramic $V F$ and/or a large piezocomposite $A R$. The method of determining the optimal values of $V F$ and $A R$ is discussed in the next section.

\section{Optimizing the 1-3 Piezocomposite Structure}

The structure of the 1-3 piezocomposite plate was optimized to maximize $k_{\mathrm{t}}$ while preventing the occurrence of the interpillar resonant mode. Figure 6 shows the flowchart of the optimization process. The algorithm was developed according to the results in $\S 3$.

First, the thickness and area of the 1-3 piezocomposite plate are determined with respect to the driving frequency. To prevent the coupling of the thickness and lateral modes of the piezocomposite plate, the width of the plate should be at least 10 times larger than the thickness. The range of $V F$ values is set to be $10-90 \%$ and that of the $A R$ values is $0.3-0.5$ to reflect the results

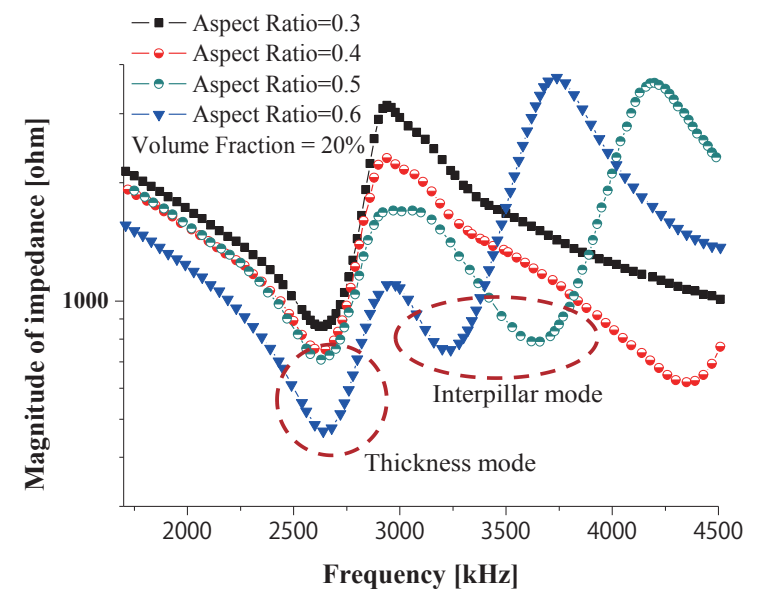

Fig. 4. (Color online) Impedance spectra as a function of $A R$.

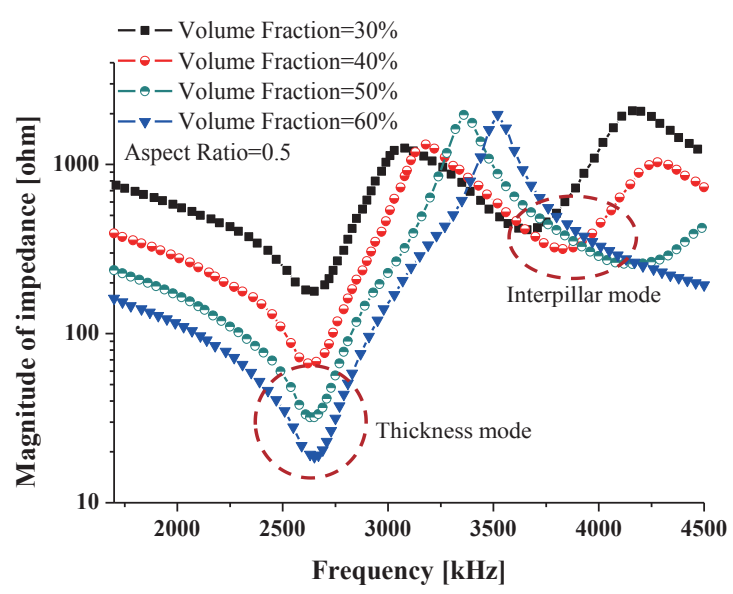

Fig. 5. (Color online) Impedance spectra as a function of $V F$. 


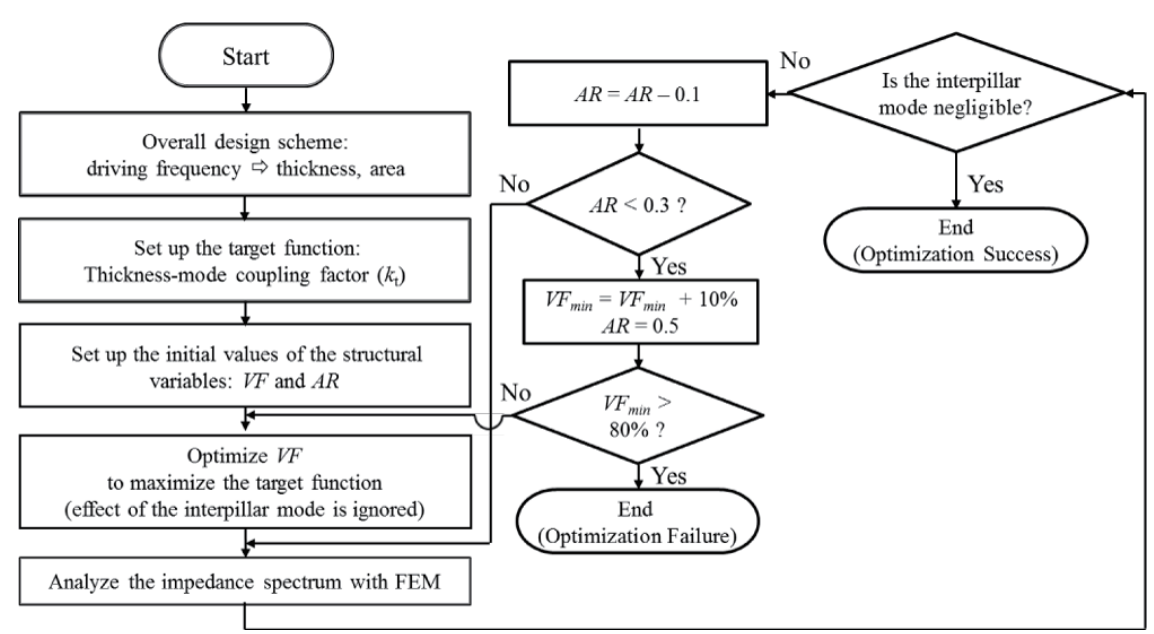

Fig. 6. Flowchart of the algorithm used to optimize the piezocomposite structure.

in $\S 3$. The initial $V F$ and $A R$ values are then set to start the optimization process. The initial $V F$ value might be an arbitrary value within the variation range, e.g., 50\%. However, the initial $A R$ value should be as large as possible within the allowed range, i.e., 0.5 , to facilitate the fabrication of the piezocomposite plate. For a given $V F$, a larger $A R$ means a larger gap between the piezoceramic pillars, which makes the dicing of the piezoceramic plate easier during the fabrication of the piezocomposite.

The effects of the structural variables $V F$ and $A R$ were analyzed with the FE model in $\S 3$. However, the FE model has a long calculation time and requires a large number of computer resources. Therefore, the optimization process in Fig. 6 was performed with analytical equations that were derived to represent $k_{\mathrm{t}}$ in terms of the structural variables. The equation for $k_{\mathrm{t}}$ was derived as a function of $V F$, as shown in Eq. (1), under the assumption that the piezocomposite plate had a pure thickness mode of vibration. ${ }^{(10-12)}$ The material constants with an upper bar in Eq. (1) represent the properties of an equivalent single-phase material that consists of the piezoceramic pillars and polymeric matrix. The $c, e$, and $\varepsilon$ parameters are the elastic stiffness, piezoelectric, and dielectric constants, respectively. The material constants with the superscript $P$ indicate the properties of the polymeric matrix, and all the other constants indicate those of the piezoceramic pillars. The superscripts $D, S$, and $E$ indicate constant electric displacement, mechanical strain, and electric fields, respectively.

$$
k_{\mathrm{t}}=\bar{e}_{33} / \sqrt{\bar{c}_{33}^{D} \bar{\varepsilon}_{33}^{S}}
$$

where

$$
\begin{gathered}
\bar{e}_{33}=V F\left[e_{33}-\frac{2(1-V F) e_{31}\left(c_{13}^{E}-c_{12}^{P}\right)}{V F\left(c_{11}^{P}+c_{12}^{P}\right)+(1-V F)\left(c_{11}^{E}+c_{12}^{E}\right)}\right], \\
\bar{c}_{33}^{D}=\bar{c}_{33}^{E}+\bar{e}_{33}^{2} / \bar{\varepsilon}_{33}^{S},
\end{gathered}
$$




$$
\bar{c}_{33}^{E}=V F\left[c_{33}^{E}-\frac{2(1-V F)\left(c_{13}^{E}-c_{12}^{P}\right)^{2}}{V F\left(c_{11}^{P}+c_{12}^{P}\right)+(1-V F)\left(c_{11}^{E}+c_{12}^{E}\right)}\right]+(1-V F) c_{11}^{P},
$$

and

$$
\bar{\varepsilon}_{33}^{S}=V F\left[\varepsilon_{33}^{E}+\frac{2(1-V F) e_{31}^{2}}{V F\left(c_{11}^{P}+c_{12}^{P}\right)+(1-V F)\left(c_{11}^{E}+c_{12}^{E}\right)}\right]+(1-V F) \varepsilon_{11}^{P} .
$$

For the initial $A R$, the $V F$ required to maximize $k_{\mathrm{t}}$ is determined by the GA. However, the interpillar mode is not considered in Eq. (1) because this equation was derived under the assumption that the piezoelectric composite has a pure thickness mode of vibration. Hence, the optimized $V F$ and $A R$ should be examined to determine if they will generate an interpillar mode. In this study, the occurrence of the interpillar mode was examined by analyzing the impedance spectrum of a modeled piezocomposite plate with the optimized $V F$ and $A R$. The impedance spectrum was calculated with the FE model over a frequency range near the thickness mode and then observed to see if any coupling with the interpillar mode occurred. If there is coupling within the frequency range, $A R$ is decreased by 0.1 to move the interpillar mode out of the frequency range. The impedance spectrum is then calculated again with the new $A R$. If coupling is still observed when $A R$ has decreased to 0.3 , the minimum $V F$ in the variation range is increased by $10 \%$ and $A R$ is reset to its initial value (0.5). That is, the variation range of $V F$ is modified to be $20-90 \%$, and the entire optimization process is repeated again with the new $V F$ range. The FE analysis is then iterated with the new $A R$ and $V F$ to examine for the occurrence of the interpillar mode. If the interpillar mode remains within the frequency range even after $A R$ reaches 0.3 and $V F$ reaches $90 \%$, the optimization process has failed. However, in all of the cases we tested, the interpillar mode was removed from the frequency range of the thickness mode when $A R$ and $V F$ were modified within the variation limits.

We checked the applicability of the optimization algorithm with a test case. The test case started with the piezocomposite structure that exhibited the impedance spectrum in Fig. 3. The spectrum shows clear coupling between the thickness and interpillar modes. The initial $V F$ and $A R$ were $25 \%$ and 0.79 , respectively, with the initial $k_{\mathrm{t}}$ of 0.43 as mentioned in $\S 2$. However, after performing the structural optimization with the algorithm in Fig. 6, the resulting impedance spectrum was improved, as shown in Fig. 7. The optimal $V F$ and $A R$ were determined to be $56.5 \%$ and 0.4 , respectively. With these values, $k_{\mathrm{t}}$ was enhanced from 0.43 to 0.67 . For underwater transducers, higher $k_{\mathrm{t}}$ means (1) higher efficiency of the transducer in converting electrical energy to acoustical energy and vice versa leading to higher sensitivity of the transducer, and (2) a wider frequency bandwidth of the transducer leading to higher resolution of the transducer, as shown in Ref. 5. High sensitivity and wide bandwidth are the two most important performance factors of underwater transducers.

The impedance spectrum in Fig. 7 shows a clear thickness mode without any coupling to interpillar modes, which confirms the efficacy of the structural optimization method presented in this paper. 


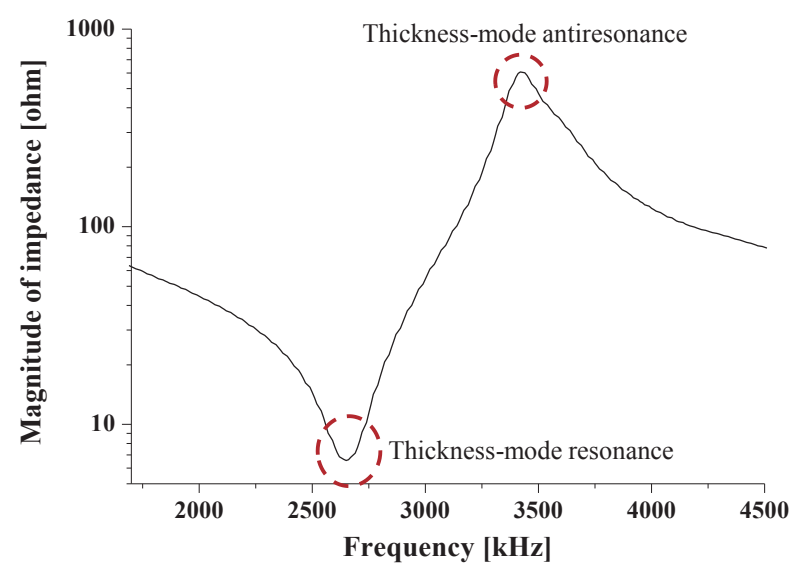

Fig. 7. (Color online) Impedance spectrum of the 1-3 piezocomposite plate whose structure was modified from that in Fig. 3 by the optimization algorithm in Fig. 6.

\section{Conclusions}

When a piezocomposite vibrates in a thickness mode, interpillar modes of vibration are likely to occur between lattice-structured piezoceramic pillars and a polymer matrix. Once generated, the interpillar mode significantly impairs the performance of the piezocomposite. Conventional methods of designing the structure of 1-3 piezocomposites have not considered these interpillar modes. Hence, we developed a new method of designing the structure of 1-3 piezocomposites that maximizes $k_{\mathrm{t}}$ while preventing the occurrence of the interpillar modes. The GA was used to optimize the structure, and an FE model was used to analyze the interpillar modes. The efficacy of the new design method was confirmed with a test case. The algorithm optimized the $A R$ and $V F$ of the piezoceramic pillars, which improved $k_{\mathrm{t}}$ from 0.43 to 0.67 while removing the coupling between the thickness and interpillar modes that was present in the initial structure.

\section{Acknowledgements}

This work was supported by the Agency for Defense Developement under the contract UD150003DD.

\section{References}

1 T. R. Gururaja, W. A. Schulze, L. E. Cross, R. E. Newnham, B. A. Auld, and Y. J. Wang: IEEE Trans. Son. Ultrason. SU-32 (1985) 481.

2 R. E. Newnham: Ferroelectrics 68 (1986) 1.

3 E. K. Akdogan, M. Allahverdi, and A. Safari: IEEE Trans. Ultrason. Ferroelectr. Freq. Control 52 (2005) 746.

4 R. E. Newnham, D. P. Skinner, and L. E. Cross: Mater. Res. Bull. 13 (1978) 525.

5 D. L. Pei and Y. Roh: Jpn. J. Appl. Phys. 47 (2008) 4003.

6 K. C. Benjamin: J. Electroceram. 8 (2002) 145.

7 X. Cheng, D. Xu, L. Lu, S. Huang, and M. Jiang: Mater. Chem. Phys. 121 (2010) 63.

8 O. Sigmund, S. Torquato, and I. A. Aksay: J. Mater. Res. 13 (1998) 1038. 
9 L. V. Gibiansky and S. Torquato: Struct. Optimization 13 (1997) 23.

10 W. A. Smith and B. A. Auld: IEEE Trans. Ultrason. Ferroelectr. Freq. Control 38 (1991) 40.

11 W. A. Smith: IEEE Trans. Ultrason. Ferroelectr. Freq. Control 40 (1993) 41.

12 J. Kim and Y. Roh: Sens. Actuators, A 206 (2014) 97.

13 B. A. Auld and Y. Wang: Proc. IEEE 1987 Ultrason. Symp., ed. B. R. McAvoy (IEEE, Denver, 1987) p. 528.

14 V. Matko and M. Milanovič: Sens. Actuators, A 220 (2014) 262.

15 V. Matko: Sensors 5 (2011) 4474.

16 A. Arnau: Sensors 8 (2008) 370.

17 C. Behling, R. Lucklum, and P. Hauptmann: Sens. Actuators, A 61 (1997) 260.

18 S. Pyo and Y. Roh: Jpn. J. Appl. Phys. 54 (2015) 07HB03-1.

19 D. Certon, F. Patat, F. Levassort, G. Feuillard, and B. Karlsson: J. Acoust. Soc. Am. 101 (1997) 2043.

20 P. Reynolds, J. Hyslop, and G. Hayward: Proc. IEEE 2003 Ultrason. Symp., ed. D. E. Yuhas (IEEE, Honolulu, 2003) p. 1650.

21 F. Craciun, L. Sorba, E. Molinari, and M. Pappalardo: IEEE Trans. Ultrason. Ferroelectr. Freq. Control 36 (1989) 50.

22 D. Robertson, G. Hayward, A. Gachagan, and V. Murray: IEEE Trans. Ultrason. Ferroelectr. Freq. Control 53 (2006) 1503.

23 C. N. Della and D. Shu: Sens. Actuators, A 140 (2007) 200.

24 A. D. Belegudu and T. R. Chandrupatla: Optimization Concepts and Applications in Engineering, ed. P. Gordon (Cambridge University Press, New York, 2014) 2nd ed., Chap. 3.

25 Z. Shen, J. Li, R. Chen, Q. Zhou, and K. K. Shung: J. Am. Ceram. Soc. 94 (2011) 1346. 